\title{
Michèle Therrien (1945-2017)
}

L'ethnographe des mots à l'écoute de la langue et de la culture inuit

Frédéric Laugrand

\section{(2) OpenEdition}

\section{Journals}

Édition électronique

URL : https://journals.openedition.org/jsa/17009

DOI : 10.4000/jsa. 17009

ISSN : 1957-7842

Éditeur

Société des américanistes

Édition imprimée

Date de publication : 15 juin 2019

Pagination : 163-174

ISBN : 978-2-902715-11-4

ISSN : 0037-9174

Référence électronique

Frédéric Laugrand, " Michèle Therrien (1945-2017) », Journal de la Société des américanistes [En ligne], 105-1 | 2019, mis en ligne le 17 juin 2019, consulté le 04 septembre 2022. URL : http:// journals.openedition.org/jsa/17009; DOI : https://doi.org/10.4000/jsa.17009 


\title{
Michèle Therrien (1945-2017). L'ethnographe des mots à l'écoute de la langue et de la culture inuit
}

\author{
Frédéric LAUGRAND \\ avec la collaboration de Marie MAUZÉ*
}

Michèle Therrien faisait l'ethnographie des mots. Traquant leurs sens et leur histoire, pas un terme ne lui échappait. Ses travaux sur la néologie (1996a et $b ; 2000 a$ et $b^{1}$ ) permettent de saisir une caractéristique majeure des sociétés inuit, à savoir leur capacité à emprunter et à s'approprier de nombreux éléments culturels exogènes sans jamais se sentir menacées, comme si les Inuit parvenaient à incorporer des nouveautés sans perdre leur identité. C'est ce que Michèle Therrien entend lorsqu'elle évoque « la dynamique de la langue inuit ». Ce sera plus tard le titre d'un livre qu'elle éditera avec Nicole Tersis (Therrien et Tersis 1996), soulignant que l'Inuktitut se caractérise par « une remarquable souplesse morphologique », ce qui lui vaut le titre de " langue à transformation » (ibid., p. 26). Et lorsque Michèle Therrien examine l'écriture inuit, celle du syllabaire introduit jadis par les missionnaires wesleyens pour faciliter la diffusion du christianisme, cette même idée des transformations dans la continuité ressurgit:

L'écrit, expérience sans précédent chez les Inuit... rappelle une expérience connue de tous: les traces de pas imprimées sur la neige et suivies. Tout comme une trace au sol, le signe de papier varie en fonction de sa direction, de son espacement, de sa forme, de son rythme. L'écrit, comme l'empreinte sur la neige, est tantôt lisible, tantôt illisible. Il est le résultat inanimé d'un mouvement antérieur du corps, que le discours, qui s'élabore autour de lui, ré-anime. (1989, p. 86)

Cette analyse que Michèle Therrien développe dans les tribunes du Journal de la Société des américanistes (1990) éclaire en quelques mots l'attachement des Inuit au syllabaire dont on sait aujourd'hui qu'il a effectivement contribué à

* F. Laugrand: Université catholique de Louvain/Département d'anthropologie, Université Laval [Frederic.Laugrand@uclouvain.be]; M. Mauzé: Laboratoire d'anthropologie sociale, Collège de France, EHESS, CNRS PSL Université de recherche [marie.mauze@collegede-france.fr].

1. Toutes les références indiquant uniquement une date renvoient à Michèle Therrien. Dans le cas contraire, les noms sont précisés. 
protéger la langue et l'identité des groupes vivant dans les régions de l'Arctique de 1'Est. Ce mode graphique partage en effet des affinités indéniables avec l'oralité. Titiqpaa, « il trace un repère » est très proche de titiraqpaa, « il écrit quelque chose », souligne l'ethnolinguiste. La trace, poursuit encore Michèle Therrien, anime la nature, elle est « une expérience à la fois pragmatique et symbolique », une ouverture, elle relie les vivants les uns par rapport aux autres, les mondes visibles et invisibles, elle crée des liens étroits avec d'autres expériences de marquage parmi lesquelles figurent la couture et le tatouage. Elle illustre bien la pensée associative des Inuit et ses débordements, car si la trace est un signe visible sur le sol, elle est inextricablement liée à une expérience sonore qui requiert une acuité auditive, ouvrant une voie royale à l'imaginaire.

Comme Jorge Luis Borges qu'elle admirait, Michèle Therrien ne manquait jamais une occasion de rappeler la puissance de la langue et de l'oralité. Ce travail débute avec son analyse du corps inuit qui traverse toute son œuvre. Passionnée par la parole, celle des humains bien entendu mais aussi celle des êtres invisibles et des animaux, elle est une des rares spécialistes du monde inuit à l'avoir écoutée et pratiquée jusqu'à son décès le 16 octobre 2017.

Sa passion pour l'ethnographie des mots et la linguistique commence en 1969, à l'issue d'un séjour d'un an au Nunavik, à Salluit en 1969, où elle occupe le poste d'enseignante et apprend la langue inuit. Ses premiers contacts avec la langue se sont faits brutalement, confie-elle, confrontée alors plus que jamais aux rivalités entre Québec et Ottawa et à leurs administrations respectives. Cette conscience de la question linguistique est restée vivante car dans une de ses lettres datée du 7 juin 1993, elle décrivait non sans humour le sentiment qu'elle avait ressenti à l'âge de 13 ans lors d'un séjour dans le Canada anglophone: « Je n'ai jamais oublié mon séjour dans les Maritimes, [que] si l'anglais s'avale mal, les homards et le paysage glissent tout seul. » Dans le Nord, c'est dans ce contexte d'affrontement entre langues dominante et dominée que Michèle Therrien s'est plongée dans l'apprentissage de l'inuktitut.

Une dizaine d'années plus tard, en 1980, sa thèse de troisième cycle Lexique du corps et champs métaphoriques (Nouveau-Québec) soutenue au Centre d'études arctiques (Paris) aboutira à la publication en 1987 d'un ouvrage fondateur devenu un classique de l'inuitologie, Le corps inuit. Dans ce livre, dont il est impossible de restituer la richesse en quelques lignes, Michèle Therrien présente l'approche ethnolinguistique dont elle se revendique, s'inspirant en autres, des perspectives de Algirdas Julien Greimas, de Jeanine Fribourg et de Geneviève Calame Griaule. Corps, langue et culture sont abordés ensemble avec la conviction que les mots et les lexiques donnent accès à des visions du monde. Dans ce cadre, le corps est un opérateur privilégié et la langue un révélateur des relations que l'homme entretient avec lui-même et son milieu (1987a, p. 21). Les proximités entre la maison de neige et le corps inuit illustrent ce point: 
Toute maison de neige possède:

- une ouverture paa qui ne désigne plus aujourd'hui la bouche mais qui prend par rapport au mot qilak (« voile du palais », « cintre de porte », " voûte de la maison ») le sens de bouche,

- deux trachées-artères tuqsuuk, soit le corridor double,

- un nez qingaq, c'est à dire le trou d'aération,

- un épi de cheveux kajjik, soit la spirale de la voûte de la maison,

- une petite base du cou manuaq, soit la marche du seuil,

- une entrée/anus itirvik. [...]

La maison, tout comme le corps, semble perçue comme un lieu d'échange. Pénètrent dans la maison les humains et les animaux à consommer, sont rejetés les morts et les détritus. La maison constitue une expérience répétitive d'entrées et de sorties. Sortir, en langue inuit, signifie également naitre : anisimajuq « il est sorti de la maison » ou « le bébé est né ». (1986a, p. 211)

Dans son ouvrage, une multitude d'autres associations sont établies, comme celle permettant de voir une continuité entre l'utérus, l'iglou et la voûte céleste.

C'est pour ma part à cette époque que j'ai rencontré Michèle pour la toute première fois, alors qu'elle enseignait l'inuktitut avec beaucoup de passion dans les petites salles de classe de l'Inalco (2 rue de Lille, à Paris). Ma formation lui doit beaucoup. C'est aussi dans ses cours que j'ai eu la chance de rencontrer des chercheurs comme Xavier Blaisel et Vladimir Randa qui intervenaient à l'occasion, mais également des artistes-peintres, des fonctionnaires, des musiciens. Les cours de Michèle Therrien rassemblaient en effet des étudiants et des auditeurs libres dont les profils étaient fort variés.

Près de quinze ans plus tard, j'ai eu la chance d'organiser avec elle deux ateliers de transmission des savoirs sur la santé auxquels des aînés comme Ilisapi Ootoova, Tipuula Qaapik Atagutsiak, Tirisi Ijjangiaq, Jaikku Pitseolak, Aalasi Joamie, Akisu Joamie, Malaija Papatsie ont participé. Les moments passés avec les aînés et les étudiantes inuit, en classe comme dans la toundra, restent gravés dans ma mémoire, tout comme ce que plusieurs aînés disaient du pouvoir des mots associés à des balles lorsqu'ils sont chargés de mauvaises intentions. Je me souviens des liens étroits que Michèle tissait avec les étudiants inuit, Susan Enuaraq, Myna Ishulutak, Nancy Kisa, Bernice Kootoo, Jeannie Shaimajuk, Julia Saimaiyuq et Eena Alivataq, mais aussi Elisapi Ikkidluak, Aatami Paniaq, Vera Arnatsiaq, Hanna Oulayoo, Mathieu Boki, Lavaani Aakulujuk, Eileen Kilabuk, Linda Apalialuk, Sheepa Nowyuk et Jane Tagak. Plusieurs sont venus ensuite enseigner la langue à Paris, d'autres continuent aujourd'hui à dispenser des cours sur la langue et la culture au sein d'associations ou dans des films: qu'on pense à Avaala de Myna Ishulutak qui a interviewé sa grand-mère et documenté ces chants que les chasseurs adressent à l'animal avant de leur infliger le coup fatal, ou encore à Inuk en colère où Aaju Piita joue un rôle clé. 
Je n'oublierai jamais non plus toutes ces journées passées ensuite à Paris avec Michèle, alors qu'elle logeait dans un petit appartement rue Charcot, à traduire mot à mot les propos des aînés. Le verbatim de ces échanges a été publié en inuktitut, puis en anglais et en français (Therrien et Laugrand 2001). Peu de gens imaginent l'énergie que cette tache a requise, en plein mois de juillet. Travaillant sans relâche, Michèle Therrien a souhaité que nous citions dans notre introduction l'image qu'Akisu Joamie avait un jour utilisée pour parler de la trop grande retenue des Inuit eu égard à la parole: " On dirait que nous les aînés, nous qui sommes les plus âgés, nous nous cachons dans le culot d'une pipe. On n'en sort que pour participer à un projet et assister à une réunion, et puis après, nous retournons à nouveau dans le culot de la pipe. [...] Il faut que nous mettions sur papier notre savoir, même si jadis il n'était transmis qu'oralement. » Pour les Inuit, comme pour Michèle, cet exercice de transcrire l'oralité s'avérait donc nécessaire et pourtant pas sans risque, mais la supériorité de l'oralité ne faisait aucun doute.

Michèle Therrien savait mieux que quiconque faire parler les mots. Plusieurs exemples illustrent ce point. Un de ses textes consacrés au baiser en offre un premier cas, alors qu'elle aborde la question des odeurs :

Les témoignages des explorateurs et des missionnaires laissaient entendre que l'odorat inuit était fort peu développé puisque capable de résister aux odeurs de graisse, de viande « avancée », d'urine, de gibier dépecé, et ce, à l'intérieur de leurs habitations bondées et peu aérées. La langue et les pratiques révèlent, tout au contraire, une extrême sensibilité aux odeurs mais celle-ci est restée incomprise devant la force d'évocation, pour ne citer qu'un exemple, du lavage des cheveux à l'urine, « cette agréable odeur de jeune fille ». (1998a, p. 207)

Un autre exemple est son analyse des rêves d'une apprentie-chamane dans laquelle elle montre bien comment le rêve qui doit toujours être partagé « est le refuge de la parole réprimée, notamment celle des enfants violés ou des adultes qui ont eu des relations sexuelles avec un non-humain » (2002a, p. 174). De ce travail, l'ethnolinguiste livre une conclusion tout à fait juste. Elle souligne que pour les Inuit, la pensée est « le seul territoire de la véritable intimité », et qu'elle doit donc ne pas être abusivement dénudée, ce qui explique qu' « il est toujours mal vu d'exprimer ouvertement ses opinions et sentiments personnels, ou de soutirer une confidence » (ibid., p. 174).

Un dernier exemple de ce travail rigoureux qui lui a été cette fois en partie commandé par le Nunavut Arctic College à Iqaluit, a été l'analyse des concepts inuit utilisés dans le domaine du droit (1997). Michèle Therrien fait ressortir l'opposition structurelle entre deux grandes catégories, celle de piqujaq - qui désigne « ce qui est demandé de faire par une personne autorisée », et concerne les droits coutumiers ou les règles sociales, la tradition inuit - et celle de maligaq - qui renvoie elle à l'idée de suivre sans en avoir le choix, une personne ou 
des idées ou des animaux, cette notion évoquant aujourd'hui directement la loi canadienne à laquelle les Inuit sont soumis. Michèle Therrien consacrera beaucoup de son énergie à éclairer les usages et les conceptions inuit, collaborant avec le juge Pierre Rousseau ou animant des émissions de radio sur ce sujet. De manière plus large, elle a suivi de très près l'histoire des relations entre Inuit et Blancs depuis le $\mathrm{XIX}^{\mathrm{e}}$ siècle. En s'appuyant sur les archives privées de la société Revillon Frères qui installe des postes de traite du Labrador à l'est du Canada jusqu'au Rocheuses et dispute le monopole de la traite des fourrures de la Compagnie de la baie d'Hudson pendant 25 ans (1901-1926), Michèle Therrien analyse dans une série d'articles (1985, 1986b, 1992, 1993, 2000c ; Therrien et Litalien 1985) la vie quotidienne dans les postes, les relations entre les chefs de poste et les familles autochtones; elle met aussi le système et les règles d'échange instaurés par les traiteurs français qui fait des autochtones pourvoyeurs de fourrure des clients consommateurs de biens européens endettés auprès de la compagnie. Elle rédigera aussi plusieurs articles et livres sur la Convention de la baie James $(1974,1978,1979)$, et plus récemment sur la naissance du Nunavut (1999a et b; 2000d), montrant à chaque fois la part active des Inuit dont elle défend corps et âme les perspectives.

L'ethnolinguiste abordera de multiples aspects des traditions inuit. Après avoir traité de la maison-corps, envisagée comme des lieux d'échanges, elle décrira de manière tout aussi remarquable la relation du chasseur à sa proie. Se plongeant cette fois dans des corpus de chants inuit, elle met en exergue la complexité des rapports humains-animaux, " l'intimité profonde qui lie l'homme au gibier ». Elle relève qu'en dépit du fait que l'homme arctique est bien un prédateur et que l'animal lui est « totalement subordonné », cette logique s'inverse dans l'imaginaire où l'homme se soumet à l'autorité des animaux qui « ne supportent pas l'insubordination des chasseurs malhabiles ou insouciants », à tel point que le chasseur doit plaire, séduire son gibier. Ce dernier s'offrira alors à lui en consentant à ce que sa dépouille soit utilisée. Un mot résume à lui seul cette relation: anginnaqtuq, qui signifie à la fois « il revient bredouille » et « sa demande n'a pas été agréée » (1987b, p. 106).

Attachée à replacer humains et animaux sur le même pied, Michèle Therrien fait remarquer que le terme de nillipuq (« émettre un son »), s'applique autant à l'humain qu'à l'animal, une série d'analogies venant confirmer cette parenté. Ainsi niksaapuq signifie à la fois le cri du lagopède et le rot de l'homme, qavaqtut, les bruits du lagopède et ceux des femmes qui jacassent; ungaa le cri du lièvre arctique blessé et les pleurs du nouveau-né, etc. Ces observations la conduisent à poser une question majeure pour l'anthropologie, « Mais qui imite qui ? » Sa réponse souligne que les humains empruntent visiblement beaucoup aux animaux qu'ils imitent. En témoignent, par exemple, ces katajjait, ces chants haletés des femmes qui reproduisent le son des oies au moment de la pariade (1987b, p. 108-109). Mais ce n'est pas tout, l'ethnolinguiste mettra en lumière 
toutes ces paroles amicales, ces demandes de clémence, ces paroles de séduction, ces mots affectueux que les chasseurs prononcent à l'endroit de leurs proies, y compris ces paroles sacrées et ces précautions langagières que les humains utilisent pour interagir avec les animaux. Bien qu'ils les chassent pour vivre, les Inuit leur reconnaissent en effet de multiples compétences dont ils ont tout à apprendre: capacité du plongeon à indiquer là où se trouvent des caribous mais aussi à prévenir de l'imminence d'une tempête, etc. Michèle Therrien conclut au « caractère indissociable de ces êtres vivants, différents et pourtant si proches l'un de l'autre » en raison des aptitudes psychiques qu'ils partagent, les animaux pouvant comme les humains penser, imaginer, choisir et parler.

C'est enfin également par la langue que Michèle Therrien a contribué de façon magistrale à comprendre le processus de remémoration et le fonctionnement de la mémoire inuit. Analysant la série Innarnik apiqsuqattarniq/Interviewing Elders à laquelle elle a participé avec le volume sur la santé (Therrien et Laugrand 2001), elle conclut que ce projet donne à voir « une mémoire en action, sollicitée dans un contexte institutionnel pour sauvegarder le contenu d'un savoir oral, mais débouchant sur l'écrit » (2002b, p. 170). Pour étayer sa démonstration, Michèle Therrien examine ce qu'elle nomme « des faits de langue » ou plus précisément « des éléments lexicaux » que les aînés mobilisent pour désigner la remémoration et la conservation, dégageant à partir de ces éléments quelques traits fondamentaux du fonctionnement de la pensée réflexive inuit. Michèle Therrien oppose ici trois radicaux, iqqauma, suqqui et ujjirusuk qui - s'ils expriment tous des modalités du passage du stade de la saillance (ce que les locuteurs ont perçu et conçu) au stade de la prégnance (la mémoire excitée) - renvoie chacun à des degrés de conservation variables et à des temporalités différentes: iqqauma référant à un passé lointain comme celui de l'enfance ou à ce qu'on parvient à se représenter, ujjirusuk à ce qui conserve sa vivacité, la part de mémoire qui se cultive et suqqui à ce qui relève du souvenir, à des données non fixées dans la mémoire. Toutes ces distinctions passent souvent inaperçues chez les ethnographes alors qu'elles livrent une quantité d'informations et permettent de saisir avec beaucoup précision la transmission des savoirs inuit (2001).

Un autre chantier qu'a abordé Michèle Therrien a été celui du silence ou plutôt de la parole silencieuse, du rire (1998b) et des paroles interdites (2008), mettant en lumière une fois de plus la spécificité de la pensée des Inuit. En définitive, elle a consacré l'essentiel de ses recherches à identifier les liens qui existent entre langue, culture et pensée inuit, trouvant sa matière première dans les langues eskaléoutes et dans l'oralité arctique, faisant ressortir de manière très originale ces liens entre les mots et les choses. Qu'on nous permette un autre exemple. Une disquette informatique, soulignait-elle, se dit nipiksaq, « ce qui est destiné à produire de la voix », d'où elle concluait: « Contrairement à notre attente, les Inuit situent l'informatique dans l'univers 
de l'oralité et non dans celui de l'écrit » (1996b, p. 33). Il faut savoir en tirer les leçons, ne pas confondre acculturation et déculturation, mais reconnaître cette faculté des Inuit à s'adapter et à « inuitiser » (ibid., p. 37), à se transformer en bénéficiant du travail sélectif de leurs épistémès ou grilles d'entendement. L'hypothèse principale qui sous-tend son travail est bien que « la conceptualisation est soumise d'une part à des contraintes logiques universelles (le temps et l'espace), d'autre part à des contraintes culturelles » qui varient selon les sociétés (ibid., p. 32). Dans cette perspective, la langue livre nécessairement des informations précieuses.

De ce point de vue, un texte remarquable et tout à fait convaincant est celui qu'elle a publié dans la revue Recherches amérindiennes au Québec sous le titre, « Corps sain, corps malade chez les Inuit, une tension entre l'intérieur et l'extérieur, entretiens avec Taamusi Qumaq » (1995). L'article offre une courte synthèse car Michèle travaillait à un manuscrit beaucoup plus complet resté inédit et intitulé Santé et maladie chez les Inuit. Façons de dire et de vivre son corps. Entretiens avec Taamusi Qumaq (1992). L'ethnolinguiste montre une fois de plus la nature analogique de la pensée des Inuit, mais également leur relation très poétique au monde qui les entoure, le corps demeurant au centre de leur univers, sorte de matrice qui gouverne leur entendement des choses. Le texte est basé sur des conversations avec Taamusi Qumaq qu'elle appréciait tout particulièrement et dont elle nous montrait l'intérêt dans ses cours, en nous faisant traduire chaque semaine les notices de son dictionnaire. Ici, Michèle Therrien met à jour des corrélations entre les concepts et leur expression linguistique, considérant les syntagmes comme les archéologues traitent leurs artefacts, « comme des témoins façonnés que l'on sonde, fouille, manipule pour en extraire les éléments constitutifs » (1995, p. 71). Par l'analyse morphologique et sémantique donc, Therrien identifie les représentations inuit du corps sain et du corps malade, distinguant le vécu individuel et la réalité culturelle. Il en résulte une discussion entre des catégories de pensée, des associations et des oppositions. Michèle Therrien distingue quatre classes de maladies (aanniaq, aanniniq, qanima et piusirluniq) qui se structurent autour de plusieurs oppositions comme celles du visible et de l'invisible, du connu et de l'inconnu, du maîtrisable et du non-maîtrisable. La maladie est sournoise, elle voyage dans le corps et lorsqu'elle est grave, sa cause est souvent liée à de l'intentionnalité. Autrement dit, elle touche les contrevenants, ceux et celles qui ne respectent pas les règles. Jadis, les chamanes étaient les grands réparateurs de ces désordres. Mais dans des pages d'une clarté remarquable, l'ethnolinguistique met également en rapport les états du corps et la relation aux éléments naturels. Un seul exemple suffira, celui des règles féminines (aunarutiit) que les Inuit associent à la fonte des glaces et de la neige (aunnaq). Michèle Therrien (ibid., p. 75) conclut très justement : 
Ces rapprochements corps/nature témoignent d'une parenté profonde entre les éléments constitutifs de l'univers et ce que l'homme possède de plus précieux, son principe vital, son nom, ses capacités intellectuelles et sa composante immortelle qui en font un tout cohérent. La langue exprime sans ambiguïté, les affinités profondes entre silatuniq (la sagesse), et sila (l'air), entre qaumaniq (le savoir, la vision chamaniques) et qau (la lumière du jour), entre tarniq (la composante immortelle de la personne) et taaq (l'obscurité).

Promue au rang de professeure des universités à l'Inalco en 2000, rattachée au Centre d'études et de recherche sur les littératures et les oralités du monde (CERLOM), Michèle Therrien a enseigné la langue et la culture des Inuit pendant quarante ans. À la fin des années 1990, elle a joué un rôle déterminant dans la collaboration que le Nunavut Arctic College a mis en œuvre avec l'Inalco, l'université Laval et l'université de Leiden. Sous la responsabilité de Susan Sammons, sociolinguiste et directrice du programme de langue et culture inuit au Nunavut Arctic College, et d'Alexina Kublu, une spécialiste reconnue des savoirs inuit, ce projet de partenariat a duré plusieurs années. Il consistait à faire venir des aînés en classe pendant plusieurs semaines afin qu'ils enseignent l'histoire et la culture aux jeunes générations. Il a donné lieu à de nombreuses publications dans plusieurs séries du Nunavut Arctic College.

Dès la fin des années 1980, et donc bien avant ces développements, Michèle Therrien a été à l'origine d'un programme d'échange très fructueux qui consistait à faire circuler des étudiants français et des Inuit, entre l'Europe et le Nunavut. Chaque année, des Inuit se rendaient ainsi à Paris et à Leiden pour y enseigner la langue et la culture et, en retour, des étudiants de l'Inalco séjournaient à Iqaluit. Plusieurs générations de chercheurs ont tiré grand profit des savoirs de ces professeurs inuit parmi lesquels figuraient Oleepeeka Ikkidluak, Jay Arnakak, Alexina Kublu, Makee Kakee, Aaju Peter, Susan Enuaraq, Myna Ishulutak, et bien d'autres. Chacun à leur manière, ces Inuit font aujourd'hui un travail remarquable pour la préservation et la transmission des savoirs au Nunavut.

Michèle Therrien a contribué à la vie intellectuelle de revues notamment Études inuit/Inuit studies et Études canadiennes où elle a siégé pendant longtemps au comité éditorial (de 2008 à 2017). Elle est une membre fondatrice de l'International Ph.D. School for Studies of Arctic Societies (IPSSAS) en 2001 et a beaucoup soutenu cette activité presque unique en son genre. En 2005, alors que le séminaire se tenait à Fairbanks, en Alaska, elle a accompagné Anne-Pascale Targé, étudiante en maîtrise au département d'anthropologie de l'Université Laval, qui était gravement malade et a été emportée quelques semaines plus tard, le 10 juillet 2005. Michèle Therrien affectionnait les séminaires que l'équipe organisait et a longtemps été secrétaire de l'organisation, ne manquant pas une occasion de faire valoir l'importance de solliciter des Inuit. L'édition de 2013 avait eu lieu à Québec et Michèle m'a souvent confié le plaisir qu'elle a eu à revoir les étudiantes du Nunavut Arctic College et à rencontrer 
pour la première fois aussi des femmes inuit, comme Lisa Koperqualuk, Betsi Annahataq, sans oublier l'ainé ahiarmiut David Serkoak. Au cours de ses dernières années, Michèle Therrien a défendu la cause des Inuit et l'enseignement de leurs langues, contribuant de façon importante avec Nicole Tersis à la constitution d'un dictionnaire sur Les langues eskaléoutes paru en 2000. Elle a œuvré au sein du CNRS (France) ou pour diverses commissions scientifiques, acceptant de vulgariser les savoirs comme l'illustre son magnifique livre de synthèse sur les Inuit paru aux Belles-lettres (2012). Michèle Therrien a enfin été l'interprète du président Jacques Chirac venu rencontrer Paul Okalik, Premier ministre du Nunavut en septembre 1999. Elle a accompagné Michel Rocard, ambassadeur de France pour les pôles arctique et antarctique, à bord du Diamant, lors d'une croisière-conférence au Groenland en 2009. Michèle Therrien n'a jamais cessé de contribuer aux débats sur le Nord. Scientifique rigoureuse, grande amatrice d'art, de musique et de poétique, elle a travaillé sur de nombreux projets d'exposition, analysant aussi des trajectoires d'individus singuliers comme celle de Glenn Gould dont elle a décrit l'expérience en partie fantasmée du Grand Nord (2007).

Michèle Therrien a travaillé avec un grand nombre de collègues universitaires et non-universitaires ainsi qu'avec de nombreux Inuit du Nunavut, du Nunavik et du Groenland. En 2006, elle a été responsable de l'organisation du $15^{\mathrm{e}}$ Congrès international des études inuit, intitulé « L'oralité au XXI ${ }^{\mathrm{e}}$ siècle. Discours et pratiques inuit », qui s'est tenu au musée du quai Branly, et dont elle a publié les actes (2009).

Exigeante dans ses cours, Michèle l'était sans aucun doute, refusant tout défaitisme de la part des étudiants face aux difficultés d'apprentissage de la langue et de l'écriture syllabique. Fervente admiratrice des Inuit, Michèle l'était radicalement, trouvant toujours de bonnes raisons d'expliquer certaines tristes réalités du Nord, comme le suicide ou la violence domestique. Elle s'intéressait à tout, à leur façon de penser le genre, de manipuler les mots et les technologies, d'affronter le cybermonde, de rire, de vivre le jour et la nuit (Galinier et al. 2010), d'affronter la politique et les grands enjeux comme les changements climatiques. Créative, pleine d'humour et pédagogue, Michèle n'hésitait pas à affronter les journalistes et les opinions. Jusque dans le métro et l'autobus qu'elle empruntait au quotidien, elle arborait sa veste à capuche en peau de phoque, fin prête à défendre à tout moment l'usage des fourrures et l'importance de la chasse, solidaire des Inuit obligés de se battre pour défendre leurs droits de chasse à la Commission européenne. Combattive et déterminée, Michèle ne reculait devant rien, n'hésitant pas comme ce fut le cas à Nuuk, en 2011, à défiler dans le cortège de la toute première Gay Pride qui avait lieu au Groenland. Humaine et généreuse, Michèle l'était profondément, donnant argent, livres et documents à qui en avait besoin pour progresser dans 
sa connaissance des Inuit et de leur langue. Déterminée et infatigable, telle était Michèle que nous ne pouvons oublier. L'œuvre de cette ambassadrice des Inuit, celle qui faisait si bien parler les mots, n'a pas fini de nous inspirer et d'animer la mémoire des Inuit.

\section{Références citées}

Collignon Béatrice et Michèle Therrien (dir.)

2009 Proceedings of the 15th Inuit Studies Conference, Orality in the 21st century: Inuit discourse and practices, Inalco, Paris, CD-ROM, http://www.inuitoralityconference.com, consulté le 07/06/2019.

Galinier Jacques, Aurore Monod-Becquelin, Guy Bordin, Laurent Fontaine, Francine Fourmaux, Juliette Roullet Ponce, Piero Salzarulo, Philippe Simonnot, Michèle THERRIEN et Iole ZILLI

2010 " Anthropology of the night: cross-disciplinary investigations », Current Anthropology, 51 (6), p. 819-847.

THERRIEn Michèle et Raymonde LitaLIEN

1985 «Archives Revillon. La traite des fourrures au $\mathrm{xx}^{\mathrm{e}}$ siècle », L'archiviste, Archives nationales du Canada, Ottawa, mai-juin 1985, 12 (3), p. 16-18.

THERrien Michèle

1974 «Études sur l'administration au Nouveau-Québec esquimau depuis 1969 », Inter-Nord, 13-14, p. 363-369.

1978 «La Convention de la baie James et du Nord Québécois », Inter-Nord, 15, p. 254-263.

1979 La conférence tripartite fédérale, provinciale et inuit, Nouveau-Québec 1970, analyse socio-politique, CNRS/EHESS (Centre d'études arctiques, Separata I), Paris.

1985 «L'entreprise Revillon Frères. Réactions euro-canadiennes, amérindiennes et inuit au commerce des fourrures », in Xavier Pons et Marcienne Rocard (dir.), Colonisations. Rencontres Australie-Canada [colloque, 15-17 mars 1984, Toulouse], Université de Toulouse-Le Mirail (Travaux de l'université de Toulouse-le-Mirail, Série B, t. 7), Toulouse, p. 19-26.

1986a «Représentations et pratiques liées à l'expérience de l'habitat chez les Inuit de l'Arctique oriental canadien. Approche ethnolinguistique ", in Ethnologie et anthropogéographie arctiques, CNRS, Paris, p. 209-215.

1986 b «elations inter-ethniques et commerce des fourrures, les carnets de voyage du capitaine Thierry Mallet, Revillon Frères, 1920-1925 », Recherches amérindiennes au Québec, 26 (4), p. 35-46.

1987a Le corps inuit. Québec arctique, Société anthropologique et linguistique de France (SELAF), Paris/Presses universitaires de Bordeaux (Arctique, 1), Bordeaux.

1987b « La parole partagée, l'homme et l'animal arctiques », Cahiers de Littérature orale, 22, p. 105-130.

1989 «Identité et forme graphique, le syllabaire des Inuit de l'Arctique oriental canadien ", L'Ethnographie, 105, 85 (1), p. 71-88. 
1990 «Traces sur la neige, signes sur le papier. Significations de l'empreinte chez les Inuit Nunavimmiut », Journal de la Société des américanistes, 76, p. 33-53.

1992 «Revillon Frères et la traite des fourrures au Canada au début du xx siècle », in La traite de la fourrure, les Français et la découverte de l'Amérique du Nord, Musée du Nouveau Monde, La Rochelle/Éditions de l'Albaron, Thononles-Bains, p. 106-137.

1993 «Membres de la direction Revillon Frères et chefs de poste: deux points de vue sur la traite de la fourrure », Études inuit/Inuit studies, 17 (2), p. 49-63.

1995 «Corps sain, corps malade chez les Inuit, une tension entre l'intérieur et l'extérieur, entretiens avec Taamusi Qumaq », Recherches amérindiennes au Québec, 20 (1), p. 71-84.

1996a «Les expériences premières et la notion de dynamique généralisée » in Nicole Tersis et Michèle Therrien (dir.), La dynamique dans la langue et de la culture inuit, Peeters (Selaf, 361 ; Arctique, 4), Paris, p. 22-41.

1996 b «éologie inuit et nouvelles traductions de la culture », Études canadiennes/ Canadian Studies, 41, p. 25-39.

1997 «Inuit concepts and notions regarding the Canadian Justice System », in Desmond Brice-Bennett (dir.), Maligaliriniup uqausiqarvingat. Legal Glossary. Glossaire juridique, Iqaluit, Interpreter/Translator Program, Nunavut Arctic College, Nunatta Campus, p. 250-275 (English/Inuktitut/French), [1st ed. 1996].

1998a «Le baiser et la chance », in Diana Rey-Hulman et Michel Boccara (dir.), Odeurs du monde. Écriture de la nuit, L'Harmattan-Inalco, Paris, p. 203-208.

1998b «Les exigences du célèbre rire inuit », in Éliane Daphy et Diana ReyHulman (dir.), Paroles à rire, Inalco (Colloques Langues O'), Paris, p. 211-222.

1999a Printemps inuit, naissance du Nunavut, préface de John Amagoalik (Jaan Amarualik), Indigène éditions, Montpellier; Prix Samuel de Champlain, Institut France-Amériques.

1999b Pour mieux comprendre le Nunavut, Sylvie Teveny et Michèle Therrien (dir.), actes [Journée Nunavut, en collaboration avec la formation continue de l'Inalco, 30 janvier 1999], Institut national des langues et civilisations orientales, avec le concours de l'Ambassade du Canada en France, Paris.

2000a «Nouvelles terminologies en inuktitut, contraintes logiques, linguistiques et culturelles ", in Nicole Tersis et Michèle Therrien (dir.), Les langues eskaléoutes, Sibérie, Alaska, Canada, Alaska, CNRS Éditions (Sciences du langage), Paris, p. 283-301.

2000 b «Les Inuit et le choix de la modernité, l'arme de la néologie », in Miura Nobutaka et Kasuya Keisuke, Les Impérialismes linguistiques hier et aujourd'hui, à l'Est et à l'Ouest, Fujiwara-shoten Publishing Company, (collaboration Maison franco-japonaise à Tokyo-université de HitotsubashiInalco), p. 238-246 [en japonais].

2000c Kakoot. Récits du pays des caribous, texte présenté et annoté par Michèle Therrien, postface ethnohistorique de Yvon Csonka, Septentrion, Québec/ Sillery; traduction de l'anglais de Glimpses of the Barren Lands de Thierry Mallet (1930, 142 p.). 
2000d «CANADA. Création d'un nouveau territoire, le Nunavut », in Universalia 2000, Encyclopédie Universalis, Paris, p. 144-148.

2001 «La question du concept Inuit qaujimajatuqangit et le bilan du jeune gouvernement du Nunavut », Études canadiennes/Canadian Studies, 52, p. 131-147.

2002a «Rêves d'une apprentie-chamane inuit», Cahiers de Littérature orale, 51 ( $\mathrm{n}^{\circ}$ spécial, Récits de rêves [Nicole Belmont, dir.]), p. 169-183.

2002 b «Ce que précise la langue inuit au sujet de la remémoration », Anthropologie et Sociétés, 26 (2-3), p. 117-135.

2007 «Glenn Gould et le Nord», in Ghyslaine Guertin (dir.), Variations sur des thèmes de Gould. Québec, Momentum, p. 25-56.

2008 Paroles interdites, Michèle Therrien (dir.), Karthala/Langues O', Paris.

2012 Les Inuit, Les Belles lettres (Guide Belles lettres des civilisations), Paris.

THerrien Michèle et Frédéric LaUgrand (dir.)

2001 Interviewing Inuit elders (Ilisapi Ootoova, Tipuula Qappik Atagutsiak, Tirisi Ijjangiaq, Jaikku Pitseolak, Aalasi Joamie, Akisu Joamie, Malaija Papatsie), Vol. 5. Perspectives on traditional health, Language and Culture Program of Nunavut Arctic College, Iqaluit; version inuktitut, sous le titre Aanniarniq aanniaqtailimaniq.

THERrIEn Michèle et Nicole TERsis (dir.)

1996 La dynamique dans la langue et la culture inuit, Peeters (Selaf, 361 ; Arctique, 4), Paris. 